\title{
Muskelkontraktionen sind ein Allheilmittel
}

\section{Ein großer Review untersuchte Präventions- und Behandlungsoptionen für chronische Krankheiten. Am allerbesten schnitt die körperliche Aktivität ab.}

_ Die Prävalenz chronischer Erkrankungen nimmt signifikant zu, vor allem außerhalb von Europa. Händeringend werden daher wirksame Präventionskonzepte gesucht. Ein Review untersuchte nun den Einfluss körperlicher Aktivität auf das Risiko, eine chronische Erkrankung zu entwickeln, und ihre mögliche Rolle in der Behandlung. Betrachtet wurden dabei Übergewicht, Typ-2-Diabetes und assoziierte metabolische Erkrankungen, arterielle Hypertonie, Gefäßerkrankungen durch arteriosklerotische Prozesse, Krebs, COPD, neurodegenerative Erkrankungen, chronisches Nierenversagen und immunvermittelte Erkrankungen.

Viele Interventionen wurden analysiert. Besonders herausgearbeitet wurden die physiologischen und pathophysiologischen Mechanismen. Am Ende kamen die Autoren zu dem Fazit, dass Interventionen, die nachhaltig die Alltags- und körperliche Aktivität in der Allgemeinbevölkerung erhöhen, allen häufigen chronischen Erkrankungen effektiv primär- und sekundärpräventiv entgegenwirken. Die absolute Stärke dieser Interventionen sei die hohe Kosteneffektivität. Die Autoren bezeichnen körperliche Aktivität als die überhaupt effektivste Intervention und fordern nachhaltige Präventionsprogramme auf Bevölkerungsebene.

- Di Raimondo D, Musiari G, Miceli G et al. Preventive and therapeutic role of muscle contraction against chronic diseases. Curr Pharm Des. 2016;22:4686-99

\section{KOMMENTAR}

Es mag abstrakt und zu simpel klingen, aber basierend auf den akribisch gesammelten Daten ist die Muskelkontraktion die Therapie mit der höchsten Evidenzbasis und der besten Kosten-Nutzen-Relation in der Behandlung und Prävention von chronischen Erkrankungen. Dabei geht es nicht um Sport, sondern um die Alltagsaktivität. Der Review mag langweilig klingen, beinhaltet aber explosives Potenzial. Macht es eigentlich Sinn, Erkrankungen kostenintensiv zu behandeln, wenn Bewegung in der Prävention nichts kostet?

Dagegen wird immer angeführt, dass Lebensstiländerungen meist nicht dauerhaft bestehen. Doch hat das z. B. im Fall der SmartphoneNutzung sehr schnell und nachhaltig funktioniert. Dabei war sicher auch das Marketing für die IT-Produkte entscheidend. Könnte man auch ein erfolgreiches Marketing für körperliche Bewegung betreiben? Gesundheitsökonomische Kalkulationen zeigen, dass dafür nur etwa ein Zehntel der Marketingausgaben der großen, internationalen Konzerne nötig wäre. Vielleicht sollten wir umdenken! Alltagsaktivität könnte als Polypille des 21. Jahrhunderts genutzt werden.

Prof. Dr. med. P. Schwarz

\section{Hier steht eine Anzeige.}

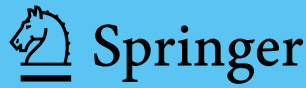

